\title{
Automatic Ascending Aorta Detection in CTA Datasets
}

\author{
Stefan C. Saur ${ }^{1}$, Caroline Kühnel ${ }^{2}$, Tobias Boskamp² ${ }^{2}$ Gábor Székely ${ }^{1}$, \\ Philippe Cattin ${ }^{1,3}$ \\ ${ }^{1}$ Computer Vision Laboratory, ETH Zurich, 8092 Zurich, Switzerland \\ ${ }^{2} \mathrm{MeVis}$ Research GmbH, 28359 Bremen, Germany \\ ${ }^{3}$ CMBE, University Basel, 4003 Basel, Switzerland \\ saur@vision.ee.ethz.ch
}

\begin{abstract}
The assessment of coronary arteries is an essential step when diagnosing coronary heart diseases. There exists a wide range of specialized algorithms for the segmentation of the coronary arteries in Computed Tomography Angiography datasets. In general, these algorithms have to be initialized by manually placing a seed point at the origins of the coronary arteries or within the ascending aorta. In this paper we present a fast and robust algorithm for the automatic detection of the ascending aorta in Computed Tomography Angiography datasets using a two-level threshold ray propagation approach. We further combine this method with an aorta segmentation and coronary artery tree detection algorithm to achieve a fully automatic coronary artery segmentation.
\end{abstract}

\section{Introduction}

The assessment of coronary arteries is an essential diagnostic tool as coronary heart diseases account for approximately $40 \%$ of deaths in both the male and female population in the European Union [1]. Computed Tomography Coronary Angiography is a frequently used modality for the detection of coronary artery diseases as it visualizes the 3D course of the coronary arteries and shows a good correlation with the gold standard, the conventional coronary angiography [2].

Automatic segmentation of the coronary arteries is desired for the diagnosis of plaques, aneurysms, or stenoses. Nowadays, a variety of specialized methods like fast marching [3], region growing [4], or model-based segmentation [5] exists for the segmentation of the coronary arteries. A detailed overview of existing segmentation algorithms for tubular structures can be found in [6]. While available algorithms perform the segmentation automatically, they still need a manual initialization in form of seed points in the origins of the coronary arteries. With the proposed ascending aorta detection, we can close this gap towards a fully automatic segmentation. The shape of the ascending aorta can be described as circular or elliptical in the slices along the $z$-direction. Thus, approaches like the Hough Transform used in [7] or the search ray approach described in [8] are suitable methods for an automatic localization of the ascending aorta. The disadvantage of the Hough Transform, however, is its limited computational efficiency. Furthermore, it is sensitive to noise and deviations of the model shape. 


\section{Methods}

The proposed procedure is based on the method described by Lorenz et al. [8] for the detection of the descending aorta. Within a fixed volume of interest (VOI) in a dataset, a search ray passes the voxels in $x$-direction (left to right), line by line (top-down), for each axial slice (Fig. 1(a)). For voxels above a predefined intensity threshold, several geometric criteria are evaluated that a candidate point for the ascending aorta must fulfill. In contrast to the descending aorta, our VOI contains more elliptical structures (e.g. right atrium, right ventricle) that fulfill the criteria used by Lorenz et al. [8]. Therefore, additional constraints were introduced to validate circularity. The detection algorithm looks as follows:

1. Trace search ray in $x$-direction while gray values stay below a given primary threshold $t h_{p}$ (Fig. 1a).

2. If primary threshold is met, continue with search ray and look for an intensity transition that falls below a secondary threshold th $h_{s}$ (Fig. 1b).

3. If transition is reached, search backwards to above $t h_{p}$ (Fig. 1b).

4. If the diameter $d_{x}$ of the found structure is within $d_{\min }$ and $d_{\max }$, compute center $p_{x}$ between both transition points and search from this center with the procedure as described above for the extension $d_{y}$ in $y$-direction. Compute center point $p_{y}$ in $y$-direction and search for extension $d_{+\pi / 4}$ and $d_{-\pi / 4}$ in direction of the two angle bisectors in the $x y$-plane (Fig. 1c).

5. Compute ratio $a=\left|d_{y} / d_{x}-1\right|$.

6. If $d_{\text {min }}<d_{i}<d_{\text {max }} \forall d_{i}, d_{i} \in\left\{d_{x}, d_{y}, d_{+\pi / 4}, d_{-\pi / 4}\right\}$ and ratio $a$ is below threshold $t h_{a}$, add $p_{y}$ to the candidate list $\mathcal{L}$.

7. Set search ray to the next line or slice, respectively, and continue with step 1 until whole VOI is processed.

Neighborhood clusters are computed out of the list $\mathcal{L}$ of candidate points. An initial cluster is defined with the first candidate point of $\mathcal{L}$. For each remaining point $p_{l}$ of $\mathcal{L}$, it is checked if a cluster already contains an element that is less than a given euclidian distance $d_{\text {clust }}$ away from $p_{l}$. If yes, the candidate point $p_{l}$ is added to this cluster. Otherwise, a new cluster is initiated. The cluster containing the most elements is taken as the representant of the ascending aorta.

In addition to Lorenz et al. we introduced the ratio $a$ and the diameters $d_{+\pi / 4}$ and $d_{-\pi / 4}$ along the direction of the two angle bisectors as extra constraints for the candidates. To speed up the detection process, the search ray is not propagated voxel by voxel, but is incremented by $\Delta_{x}, \Delta_{y}$, and $\Delta_{z}$, in $x$-, $y$-, and $z$-direction, respectively. A priori anatomical knowledge about the expected position of the ascending aorta is used to define the VOI.

We used 100 computed tomography angiography (CTA) datasets (standard cardiac protocol, Siemens Definition) to fix a set of parameters that minimizes the number of false detections: $\Delta_{x}=1 \mathrm{~mm}, \Delta_{y}=5 \mathrm{~mm}, \Delta_{z}=1 \mathrm{~mm}, t h_{p}=$ $180 \mathrm{HU}, t h_{s}=100 \mathrm{HU}, d_{\min }=20 \mathrm{~mm}, d_{\max }=70 \mathrm{~mm}, d_{\text {clust }}=5 \mathrm{~mm}$ (isotropic), $t h_{a}=0.25, \mathrm{VOI}_{x}=0-0.75, \mathrm{VOI}_{y}=0.25-0.70, \mathrm{VOI}_{z}=0.50-1.00$. The VOI is given as a percentage of the total dataset extension in the corresponding direction whereby the $z$-direction is defined in cranial direction. 
As a benchmark for the proposed algorithm, we used the method developed by Hennemuth et al. [9] to segment the ascending aorta and to detect voxel clusters at the coronary artery origins. Initialized with the detected seed point in the ascending aorta, the algorithm relies on a statistical analysis of areas segmented by a $2 \mathrm{D}$ region growing algorithm to segment the ascending aorta in caudal direction. The method automatically stops at the aortic valve and detects the origins of the coronary arteries. As the focus of this work was on the automatic detection of the ascending aorta, we did not evaluate the performance of different coronary artery segmentation approaches, although it is possible to combine the presented method with any algorithm that needs a point within the ascending aorta or the origins of the coronary arteries for its initialization.

For the initialization of the ascending aorta segmentation, a cluster point has to be selected that is sufficiently above the aortic valve and below the aortic arch. We sort the points of the largest cluster by their $z$-coordinate such that the first point in the list is closest to the aortic valve. The cluster point at the $90 \%$ quantile of the sorted list is finally selected as the seed point for the following segmentation process. The ground truth was defined by three experienced readers who manually placed a seed point within the ascending aorta for all datasets. Each reader - blinded to the seed points of the others - was instructed to mark a point within the ascending aorta well above the aortic valve and below the aortic arch. The seed point was then used as an initialization for the ascending aorta segmentation method as described above.

\section{$3 \quad$ Results}

We evaluated the presented method on 297 CTA datasets from different Siemens CT scanners (Definition, Sensation 64, Sensation Cardiac 64, Sensation 16) whereby the datasets for the parameter optimization were not included in this

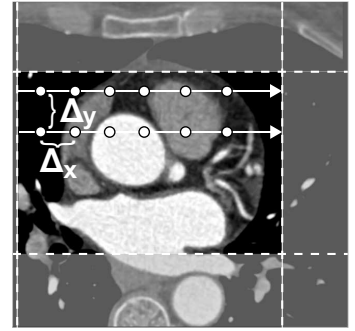

(a) VOI and ray propagation

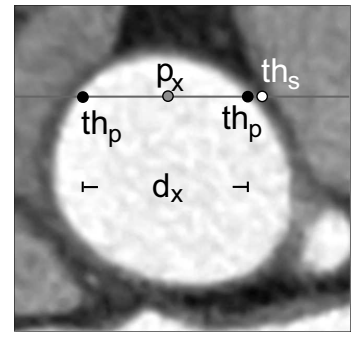

(b) Extension in $x$-direction

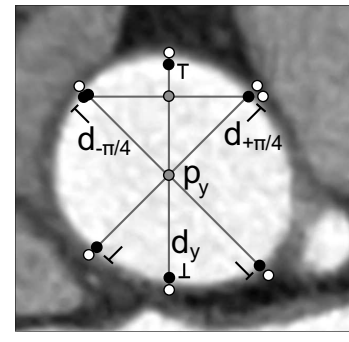

(c) Candidate point $p_{y}$

Fig. 1. Detection of the ascending aorta. Within a predefined volume of interest (VOI), search rays are propagated (a) following a two-level threshold approach to compute the extension in $x$-direction (b) and afterwards in $y$-direction as well as in direction of the two angle bisectors (c). If geometric constraints are fulfilled, the point $p_{y}$ is added to the list of ascending aorta candidates. 
Table 1. As a benchmark, we initialized an ascending aorta segmentation and coronary artery detection algorithm for both automatically detected and manually placed seed points. We evaluated the successful segmentation of the ascending aorta down-to the aortic valve as well as the detection of the left and right coronary artery tree (CAT) origin, respectively. Values are given as successful number/total number (percentage)

\begin{tabular}{lllll}
\hline & $\begin{array}{l}\text { Detection of } \\
\text { ascending aorta }\end{array}$ & $\begin{array}{l}\text { Segmentation of Detection of } \\
\text { ascending aorta }\end{array}$ & $\begin{array}{l}\text { Detection of } \\
\text { left CAT origin }\end{array}$ & right CAT origin \\
\hline Our method 288/297(97.0\%) & $284 / 288(98.6 \%)$ & $261 / 284(91.9 \%)$ & $263 / 284(92.6 \%)$ \\
Reader 1 & $297 / 297(100.0 \%)$ & $292 / 297(98.3 \%)$ & $270 / 292(92.5 \%)$ & $271 / 292(92.8 \%)$ \\
Reader 2 & $297 / 297(100.0 \%)$ & $296 / 297(99.7 \%)$ & $275 / 296(92.9 \%)$ & $279 / 296(94.3 \%)$ \\
Reader 3 & $297 / 297(100.0 \%)$ & $295 / 297(99.3 \%)$ & $274 / 295(92.9 \%)$ & $273 / 295(92.5 \%)$ \\
\hline
\end{tabular}

evaluation. The in-plane resolution ranged from $0.236-0.512 \mathrm{~mm}$ and the slice thickness varied between $0.3-0.8 \mathrm{~mm}$. Each slice contained $512 \times 512$ voxels and the number of slices varied between 150 and 591 .

A seed point was correctly placed in the ascending aorta in 288 (97.0\%) datasets. For 9 datasets, the aorta could not be detected and a point within the right ventricle (8 times) or descending aorta (once) was chosen by the algorithm. In these cases, the second largest cluster represented the ascending aorta. The mean detection time for the ascending aorta was $0.30 \mathrm{~s}$ and ranged from 0.13$0.77 \mathrm{~s}$ on a Pentium 4 with $3 \mathrm{GHz}$ and $2 \mathrm{~GB}$ RAM.

In $98.6 \%$ of the cases where our automatic detection succeeded, the aorta could be segmented down to the aortic valve. With this correct ascending aorta segmentation, a detection rate of $91.9 \%$ for the left coronary artery tree (CAT) origin and $92.6 \%$ for the right CAT origin could be achieved.

Based on different placed seed points by the readers, the aorta segmentation rate varied between $98.3 \%$ and $99.7 \%$ which led to a detection rate of $92.5 \%$ $92.9 \%$ for the left and $92.5 \%-94.3 \%$ for the right CAT origin, respectively. A detailed comparison of the results for the segmentation of the ascending aorta and the detection of the origins for the left and right CAT is listed in Table 1.

\section{Discussion and Conclusion}

We presented a fast and robust algorithm for the automatic detection of the ascending aorta in CTA datasets using a two-level threshold ray propagation approach. The method has been tuned on 100 CTA datasets and has been successfully evaluated on 297 scans from different CT devices. A detection rate of $97.0 \%$ confirms the robustness of the presented method. Figure 2 exemplarily shows four detected aortas. In all 9 cases where the largest candidate cluster did not represent the ascending aorta, it was identified by the second largest cluster. Thus, our future work will focus on the definition of additional features for the two largest clusters to further improve the detection rate.

With a successfully detected ascending aorta, the performance of the aorta segmentation and coronary artery tree origin detection was within the variability 
Fig. 2. Screenshots of four different datasets with varying contrast agent distribution and anatomical shape: In $97.0 \%$ of all cases, our presented algorithm automatically found a point within the ascending aorta (black circle)
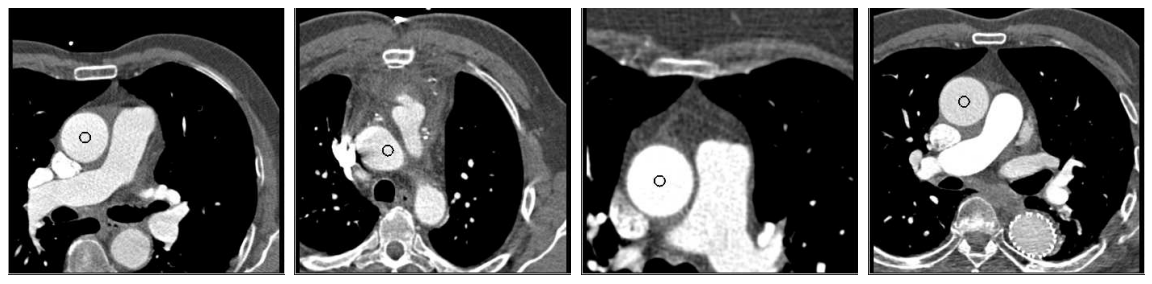

range given by the results of manually placed seed points. Hence, the developed method is suitable to speed up the diagnostic process by automatically presegmenting the coronary arteries.

Therefore, our method can close the gap to a fully automatic vessel segmentation by combining the developed ascending aorta detection with an aorta and coronary artery tree segmentation algorithm.

Acknowledgement. The authors thank the radiology departments from the Universitätsspital Zürich, Universitätsklinikum Erlangen, Radiological University Hospital Tübingen, University Hospital of Munich, PUMC Hospital and PLA Hospital in Beijing for providing the CTA datasets. This work has been supported by the CO-ME/NCCR research network of the Swiss National Science Foundation (http://co-me.ch).

\section{References}

1. Jougla E. Health Statistics - Atlas on mortality in the European Union. European Communities; 2003.

2. Leschka S, Alkadhi H, Plass A, et al. Accuracy of MSCT coronary angiography with 64-slice technology: First experience. Eur Heart J. 2005;26(15):1482-7.

3. Lorenz C, Renisch S, Schlathölter T, et al. Simultaneous segmentation and tree reconstruction of the coronary arteries in MSCT images. Proc SPIE. 2003;5031(1):167-77.

4. Boskamp T, Rinck D, Link F, et al. New vessel analysis tool for morphometric quantification and visualization of vessels in CT and MR imaging sata sets. Radiographics. 2004;24(1):287-97.

5. Wörz S, Rohr K. Segmentation and quantification of human vessels using a 3-D cylindrical intensity model. IEEE Trans Image Process. 2007;16(8):1994-2004.

6. Kirbas C, Quek F. A review of vessel extraction techniques and algorithms. ACM Computing Surveys. 2004;36(2):81-121.

7. Kovacs T, Cattin P, Alkadhi H, et al. Automatic segmentation of the vessel lumen from 3D CTA images of aortic dissection. Proc BVM. 2006; p. 161-5.

8. Lorenz C, Lessick J, Lavi G, et al. Fast automatic delineation of cardiac volume of interest in MSCT images. Proc SPIE. 2004;5370(1):456-66.

9. Hennemuth A, Boskamp T, Fritz D, et al. One-click coronary tree segmentation in CT angiographic images. Proc CARS. 2005;1281:317-321. 